\title{
Spontaneous lateral atomic recoil force close to a photonic topological material
}

\author{
S. Ali Hassani Gangaraj, ${ }^{1, *}$ George W. Hanson, ${ }^{2, \dagger}$ Mauro Antezza, ${ }^{3,4, \ddagger}$ and Mário G. Silveirinha ${ }^{3,5,8}$ \\ ${ }^{1}$ School of Electrical and Computer Engineering, Cornell University, Ithaca, New York 14853, USA \\ ${ }^{2}$ Department of Electrical Engineering, University of Wisconsin-Milwaukee, 3200 North Cramer Street, Milwaukee, Wisconsin 53211, USA \\ ${ }^{3}$ Laboratoire Charles Coulomb (L2C), UMR 5221 CNRS-Université de Montpellier, F-34095 Montpellier, France \\ ${ }^{4}$ Institut Universitaire de France, 1 rue Descartes, F-75231 Paris Cedex 05, France \\ ${ }^{5}$ Instituto Superior Técnico, University of Lisbon and Instituto de Telecomunicações, Torre Norte, \\ Avenida Rovisco Pais 1, P-1049-001 Lisbon, Portugal
}

(Received 13 November 2017; revised manuscript received 24 April 2018; published 14 May 2018)

\begin{abstract}
We investigate the quantum recoil force acting on an excited atom close to the surface of a nonreciprocal photonic topological insulator (PTI). The main atomic emission channel is the unidirectional surface plasmon propagating at the PTI-vacuum interface, and we show that it enables a spontaneous lateral recoil force that scales at short distances as $1 / d^{4}$, where $d$ is the atom-PTI separation. Remarkably, the sign of the recoil force is polarization and orientation independent, and it occurs in a translation-invariant homogeneous system in thermal equilibrium. Surprisingly, the recoil force persists for very small values of the gyration pseudovector, which, for a biased plasma, corresponds to very low cyclotron frequencies. The ultrastrong recoil force is rooted in the quasihyperbolic dispersion of the surface plasmons. We consider both an initially excited atom and a continuous pump scenario, the latter giving rise to a steady lateral force whose direction can be changed at will by simply varying the orientation of the biasing magnetic field. Our predictions may be tested in experiments with cold Rydberg atoms and superconducting qubits.
\end{abstract}

DOI: 10.1103/PhysRevB.97.201108

The force on neutral atoms or nanoparticles due to electromagnetic fields is an important tool in atomic control and manipulation (e.g., in optical trapping for ultracold gases [1-3], optical tweezers [4,5], etc.). Fluctuations of the electromagnetic field, of both quantum and thermal origin, play an important role in this context. They explain the presence of the Casimir-Polder forces acting on atoms located close to the surface of a body [6,7]. For planar surfaces the CasimirPolder force is along the normal direction and can be either attractive or repulsive [8-13]. The normal component of the force has been extensively investigated both theoretically and experimentally (see Ref. [14] and references therein).

For excited systems (e.g., an excited or driven atom) it is also possible to have nonzero lateral forces, even when the material surface is smooth and translation invariant. Such lateral forces have been studied both in classical and in quantum scenarios, e.g., when a polarizable nanoparticle is near an anisotropic substrate with a tilted axis [15], when linearly polarized light illuminates a chiral particle [16], when the emitter has circular polarization $[17,18]$, or in moving systems [19,20]. These studies reveal interesting possibilities to tune and sculpt the surface-atom interactions.

The discovery of topological light states is perhaps the most exciting development in photonics in recent years. Starting with the seminal studies of Haldane and Raghu [21,22], it

\footnotetext{
*ali.gangaraj@gmail.com

†george@uwm.edu

${ }^{\ddagger}$ mauro.antezza@umontpellier.fr

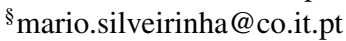

has been shown that some nonreciprocal photonic platforms are inherently topological [23-25], and thereby may enable the propagation of unidirectional topologically protected and scattering-immune edge states. More recently, it has been shown that the concepts of topological photonics can be extended to electromagnetic continua, with no intrinsic periodicity [26-31]. The consequences of topological properties in the context of quantum optics have been little explored, with a few exceptions [32-34].

In this Rapid Communication, we investigate a mechanism that enables a lateral recoil force due to the spontaneous emission features of atoms close to a photonic topological insulator (PTI) surface. In an environment invariant along the $j$-coordinate axis, the lateral quantum recoil force $\mathcal{F}_{j}$ associated with a spontaneous decay process generally vanishes since the emission occurs in random directions. For particular polarizations of the emitter (e.g., circular polarization), the lateral force may be nonzero due to the interference of the dipole and its image [18,35]. However, in systems formed by reciprocal media, the sign of the lateral force is invariably polarization dependent $[18,35]$.

In contrast, here we demonstrate that the PTI's unidirectional light states create the opportunity for unusual optical manipulations of two-level quantum systems with a strongly asymmetric, and tunable, lateral optical force in a laterally invariant environment, with the sign of the force independent of the polarization state and of the atom orientation.

We first consider a two-level system (called the "atom" in the following) in a general inhomogeneous environment. In the dipole approximation, the optical force operator is $\hat{\mathcal{F}}_{i}=\hat{\mathbf{p}}$. $\partial_{i} \hat{\mathbf{E}}, i=x, y, z$ [5]. The hat indicates that the relevant quantity 
represents a quantum operator. Here, $\hat{\mathbf{p}}$ is the electric dipole operator for the two-level atom. By solving the Heisenberg equations with the Markov approximation, it is possible to show that the expectation of the optical force is given by [36]

$$
\mathcal{F}_{i}(t)=\left\langle\hat{\mathcal{F}}_{i}\right\rangle=\rho_{e e}(t) \mathcal{F}_{R, i}+\left[1-2 \rho_{e e}(t)\right] \mathcal{F}_{C, i}
$$

with $\rho_{e e}(t)$ representing the probability of the atom being in the excited state,

$$
\mathcal{F}_{R, i}=2 \operatorname{Re}\left\{\left.\tilde{\gamma}^{*} \cdot\left[-i \omega \partial_{i} \mathbf{G}\left(\mathbf{r}, \mathbf{r}_{0} ; \omega\right)\right]\right|_{\substack{\omega=\omega_{0}+i 0^{+} \\ \mathbf{r}=\mathbf{r}_{0}}} \cdot \tilde{\gamma}\right\}
$$

is the resonant component of the force, and $\partial_{i}=\hat{\mathbf{u}}_{i} \cdot \nabla_{\mathbf{r}}$. Here, $\omega_{0}$ is the atomic transition frequency, $\tilde{\gamma}=\left[\begin{array}{ll}\gamma & 0\end{array}\right]^{T}$ is a six-vector, and $\gamma$ is the dipole matrix element. Moreover, $\mathcal{F}_{C}=-\nabla_{\mathbf{r}_{0}} \mathcal{E}_{C}$ is the usual Casimir-Polder force for the ground state [36], which vanishes for planar surfaces along laterally invariant directions.

The force is written in terms of the system Green's function $\mathbf{G}\left(\mathbf{r}, \mathbf{r}_{\mathbf{0}} ; \omega\right)($ a $6 \times 6$ tensor $)$, defined in the companion article [36], with $\mathbf{r}_{0}$ the position of the atom. Only the scattering part of the Green's function needs to be considered, because by symmetry the self-field does not contribute to the force [36]. For simplicity, we neglect the effect of thermal photons, which is acceptable when $\hbar \omega_{0}$ is higher than the average thermal photon energy $k_{B} T \ll \hbar \omega_{0}$ and when $d \ll \lambda_{T}$, with $\lambda_{T}=$ $h c / k_{B} T$ the thermal wavelength and $d$ the distance between the atom and the macroscopic body. Equation (1) generalizes the theory of Refs. [37,38] to arbitrary reciprocal or nonreciprocal and possibly bianisotropic systems.

Next, we turn to the geometry of interest, a z-stratified electromagnetic environment, invariant to translations along the $\alpha=x, y$ directions. Since the lateral force due to the zero-point energy fluctuations vanishes, $\mathcal{F}_{C, \alpha}=0$, the lateral force is determined uniquely by the resonant term $\mathcal{F}_{\alpha}(t)=\rho_{e e}(t) \mathcal{F}_{R, \alpha}$. We prove in Ref. [36] that in the limit of vanishing material loss, $\mathcal{F}_{R, \alpha}$ can be written in terms of the electromagnetic modes $\mathbf{F}_{n \mathbf{k}}$ of the environment as

$\mathcal{F}_{R, \alpha}=\operatorname{Re}\left(i \pi \sum_{\omega_{n \mathbf{k}}>0} \omega_{n \mathbf{k}} \tilde{\boldsymbol{\gamma}}^{*} \cdot \partial_{\alpha} \mathbf{F}_{n \mathbf{k}} \otimes \mathbf{F}_{n \mathbf{k}}^{*} \cdot \tilde{\boldsymbol{\gamma}} \delta\left(\omega_{n \mathbf{k}}-\omega_{0}\right)\right)$,

where $\mathbf{F}_{n \mathbf{k}}=\left(\begin{array}{ll}\mathbf{E}_{n \mathbf{k}} & \mathbf{H}_{n \mathbf{k}}\end{array}\right)^{\mathrm{T}}$ and $\omega_{n \mathbf{k}}$ is the oscillation frequency of the mode $n \mathbf{k}$. The above result fully takes into account material dispersion. In the following, we consider the recoil force on an atom in vacuum close to the interface with a topological material, e.g., a gyrotropic medium [28-30,32,39], as shown in Fig. 1(a).

As an example, we suppose the region $z>0$ is a vacuum, and that the region $z<0$ is filled with a gyrotropic material with permittivity

$$
\boldsymbol{\varepsilon}=\varepsilon_{0}\left(\varepsilon_{t} \mathbf{I}_{t}+\varepsilon_{a} \hat{\mathbf{y}} \hat{\mathbf{y}}+i \varepsilon_{g} \hat{\mathbf{y}} \times \mathbf{I}\right),
$$

where $\mathbf{I}_{t}=\mathbf{I}-\hat{\mathbf{y}} \hat{\mathbf{y}}$, with $\varepsilon_{g}$ being the magnitude of the gyration pseudovector. For the gyrotropic medium we consider a magnetized plasma (e.g., InSb $[40,41])$. For a static bias magnetic (a)

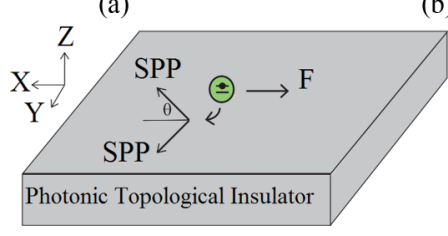

(c)
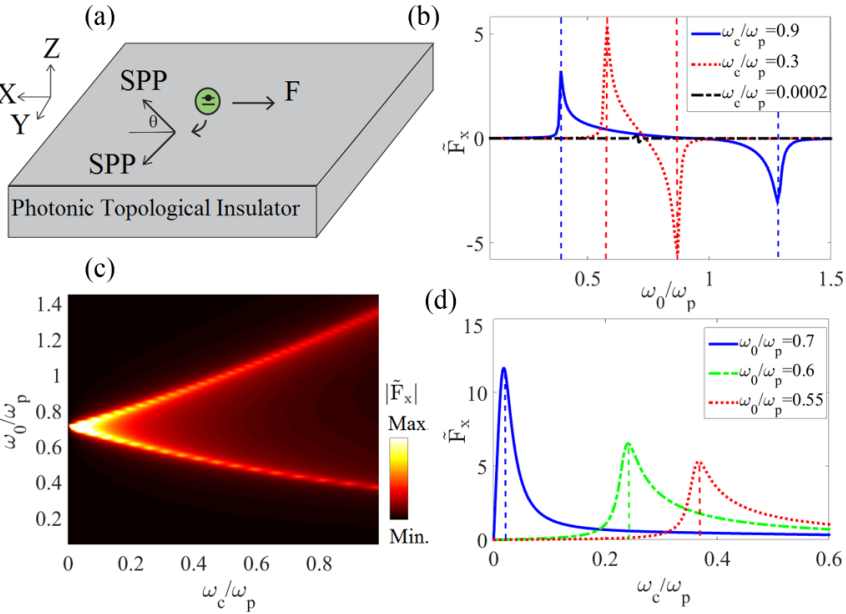

b)

FIG. 1. (a) The geometry under investigation. The green dot indicates a two-level atom above an interface between a PTI substrate and vacuum. (b) Recoil force as a function of frequency obtained from the exact solution (2). The vertical dashed lines indicate $\omega_{0}=\omega_{ \pm}$here and in (d). (c) Absolute value of the recoil force as a function of atomic transition frequency and plasma bias. (d) Recoil force as a function of plasma bias for several transition frequencies. The atom is located $d=0.01 c / \omega_{p}$ above the interface in the vacuum region.

field along the $+y$ axis, the permittivity components are [42]

$$
\begin{aligned}
& \varepsilon_{t}=1-\frac{\omega_{p}^{2}(1+i \Gamma / \omega)}{(\omega+i \Gamma)^{2}-\omega_{c}^{2}}, \\
& \varepsilon_{a}=1-\frac{\omega_{p}^{2}}{\omega(\omega+i \Gamma)}, \quad \varepsilon_{g}=\frac{1}{\omega} \frac{\omega_{c} \omega_{p}^{2}}{\omega_{c}^{2}-(\omega+i \Gamma)^{2}} .
\end{aligned}
$$

Here, $\omega_{p}$ is the plasma frequency, $\Gamma$ is the collision rate associated with damping (taken to be $\left.\Gamma=0.015 \omega_{p}\right), \omega_{c}=$ $-q B_{0} / m>0$ is the cyclotron frequency, $q=-e$ is the electron charge, $m$ is the electron effective mass, and $B_{0}$ is the static bias.

In the quasistatic lossless limit, the natural modes have

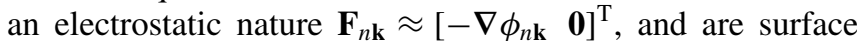
plasmon polariton (SPP) waves. In Ref. [36], it is shown that the SPP resonances have the dispersion $\omega_{\mathbf{k}}=\omega_{\theta}$ with $2 \omega_{\theta}=$ $\omega_{c} \cos (\theta)+\sqrt{2 \omega_{p}^{2}+\omega_{c}^{2}\left[1+\sin ^{2}(\theta)\right]}$. Here, $\theta$ represents the angle of the SPP wave vector $\mathbf{k}$ (parallel to the interface) and the $x$ axis. In general, we have $\omega_{-}<\omega_{\theta}<\omega_{+}$with $\omega_{ \pm}=\omega_{\theta=0 / \pi}$. The quasistatic solution describes the resonant SPP waves with large $\mathbf{k}$, which are the most influential for the lightmatter interactions. Different from the unbiased case $\left(\omega_{c}=0\right)$, for which the SPP resonance is direction independent, $\omega_{\theta}=$ $\omega_{p} / \sqrt{2} \equiv \omega_{\text {spp }}$, for a biased plasma the emitted plasmons are launched along preferred directions of space [see Fig. 1(a), and Fig. 2(a), which shows the angle of the SPP wave vector $\mathbf{k}$; a further discussion of the angular dependence of the SPP is provided in Ref. [36]]. Intuitively one may expect that the sign of the optical force $\mathcal{F}_{x}$ is contrary to the ( $x$ component) of the wave vector of the SPPs excited by the atom, i.e., the SPPs that satisfy $\omega_{ \pm \theta_{0}}=\omega_{0}$.

This heuristic argument is confirmed by a detailed calculation of the force (3) that gives $\mathcal{F}_{\alpha}=\mathcal{F}_{0} \tilde{\mathrm{F}}_{\alpha}(\alpha=x, y)$, with $\mathcal{F}_{0}=3|\gamma|^{2} /\left(16 \pi d^{4} \varepsilon_{0}\right)$ a normalizing factor, and $\tilde{\mathrm{F}}_{\alpha}$ a 
(a)

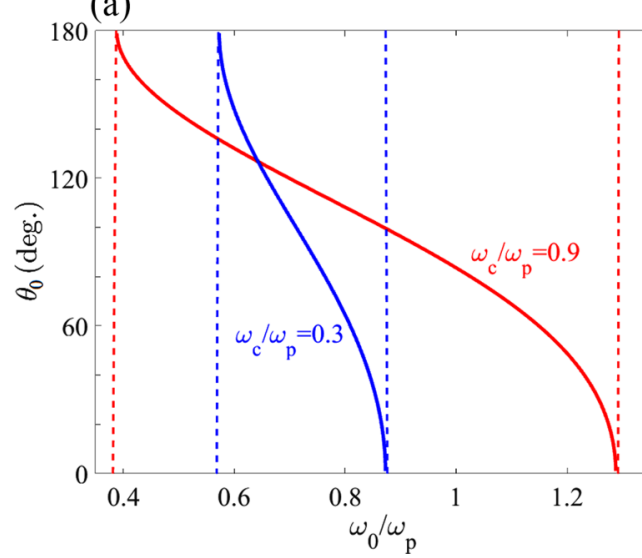

(b)

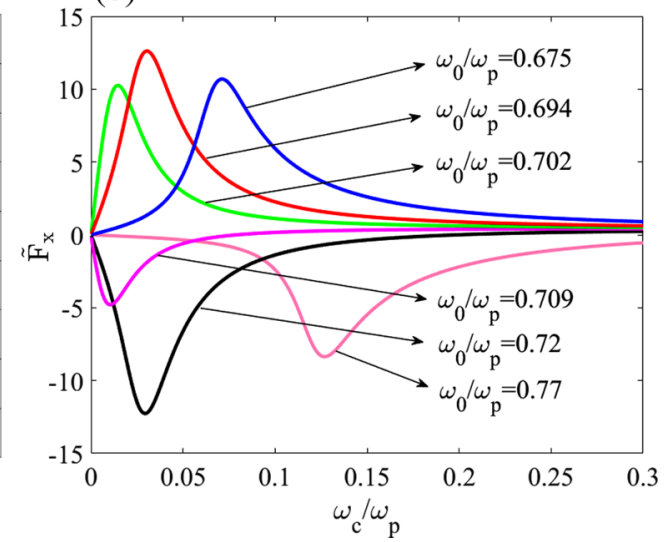

FIG. 2. (a) SPP wave-vector angle $\theta_{0}$ as a function of the atomic transition frequency for $\omega_{c} / \omega_{p}=0.4$. The vertical dashed lines indicate $\omega_{0}=\omega_{ \pm}$. (b) Recoil force as a function of cyclotron frequency for several transition frequencies. The atom is located $d=0.01 c / \omega_{p}$ above the interface in the vacuum region.

dimensionless parameter given by [36]

$$
\begin{aligned}
& \tilde{\mathrm{F}}_{x}=-\left.\rho_{e e}(t) \frac{\omega_{\theta} a_{\theta} \cos \theta}{\left|\partial_{\theta} \omega_{\theta}\right|}\right|_{\theta=\theta_{0}} \frac{1}{2}\left(\Gamma_{+, \theta_{0}}+\Gamma_{+,-\theta_{0}}\right), \\
& \tilde{\mathrm{F}}_{y}=-\left.\rho_{e e}(t) \frac{\omega_{\theta} a_{\theta} \sin \theta}{\left|\partial_{\theta} \omega_{\theta}\right|}\right|_{\theta=\theta_{0}} \frac{1}{2}\left(\Gamma_{+, \theta_{0}}-\Gamma_{+,-\theta_{0}}\right),
\end{aligned}
$$

where $a_{\theta}>0$ and $\Gamma_{+, \theta} \geqslant 0$ are defined in the Supplemental Material [43]. Therefore, the recoil force scales as $1 / d^{4}$, where $d$ is the distance between the atom and the interface. In Ref. [36] it is shown that the quasistatic force expression (6) yields results virtually identical to the exact result (2). In this Rapid Communication, we focus on $\mathcal{F}_{x}$, as the sign of the other lateral force component $\mathcal{F}_{y}$ is polarization dependent (and for a vertical dipole, $\mathcal{F}_{y}=0$ by symmetry).

Clearly, the quasistatic result shows that the lateral force is mainly determined by the plasmons that propagate with the wave vector directed along either $\theta=\theta_{0}$ or $\theta=-\theta_{0}$, and the sign of the force $\mathcal{F}_{x}$ is opposite of the sign of $\cos \left(\theta_{0}\right)$. The force is therefore antiparallel to the wave vector of the emitted plasmons, independent of the dipole polarization. This indicates that the momentum transfer is determined by the Minkowski momentum (parallel to the wave vector), rather than by the Abraham momentum (parallel to the Poynting vector, or, equivalently, to the group velocity) [44-48]. Since in a gyrotropic half space the directions of the plasmon wave vector and group velocity are generally different, this suggests that light-matter interactions at the quantum level are determined by the canonical (Minkowski) momentum of light, rather than by the kinetic (Abraham) momentum [45-47].

Figure 1(b) shows the normalized force as a function of $\omega_{0}$ for different bias strengths, calculated with the exact Green's function solution (2) for an atom with $\gamma=\gamma \hat{\mathbf{z}}$ [36]. The recoil force can be controlled by changing the magnetic bias strength, with the force existing primarily in the frequency interval $\omega_{-}<\omega_{0}<\omega_{+}$. Furthermore, the sign of the recoil force can be flipped simply by flipping the bias field $\left(\omega_{c}<0\right)$.

The two prominent observations from Fig. 1(b) are that the recoil force changes sign as $\omega_{0}$ sweeps the interval $\left(\omega_{-}, \omega_{+}\right)$, and that the recoil force is largest for $\omega_{0}=\omega_{ \pm}$, denoted by the vertical dashed lines in the figure. Both effects can be understood from conservation of momentum; the angle of the SPP wave vector $\mathbf{k}$ (the main emission channel) is shown in Fig. 2(a), where it can be seen that for $\omega_{0}=\omega_{-}, \theta_{0}=180^{\circ}$ ( $-x$ direction), resulting in a positive lateral force along $x$, whereas for $\omega_{0}=\omega_{+}, \theta_{0}=0^{\circ}$ ( $+x$ direction), leading to a negative lateral force. Similarly, the recoil force will be largest for $\omega_{0}=\omega_{ \pm}$, since $\tilde{\mathrm{F}}_{x}$ is maximized when the emitted SPP is along the $\pm x$ axis.

A further aspect of the SPPs is that they form beamlike far-field patterns due to the hyperbolic nature of the material. Figure 3 shows the SPP equifrequency contour (EFC) for the case of $\omega_{0} / \omega_{p}=0.7$ for various values of $\omega_{c} / \omega_{p}$. For (a)
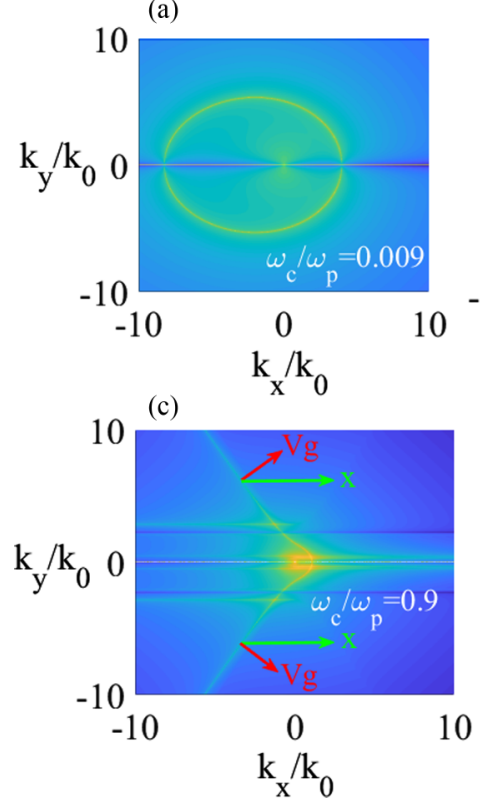

(b)

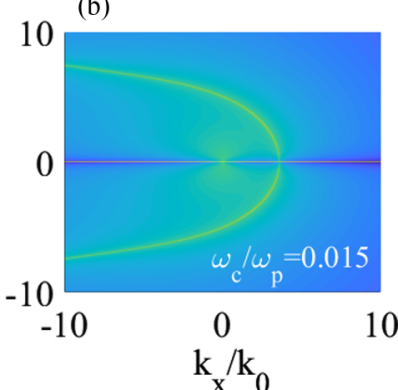

(d)

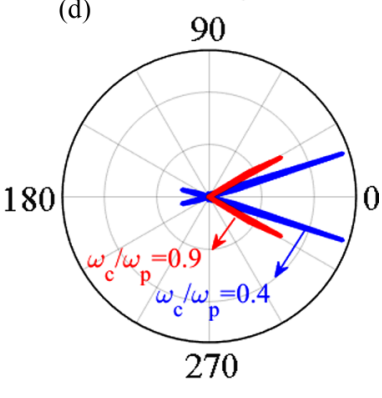

FIG. 3. (a)-(c) Equifrequency contours for the SPP at the interface of a magnetized plasma and vacuum, for different bias strengths, with $\omega / \omega_{p}=0.7$ and $k_{0}=\omega / c$. The EFC is a density plot of the exact SPP dispersion equation [36]. The red arrows in (c) indicate the direction of SPP energy flow. (d) SPP far-field pattern (radiated by a classical dipole polarized along $z$ ) for two bias values. 
(a)

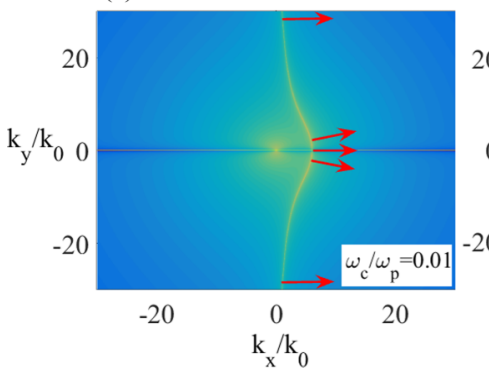

(b)

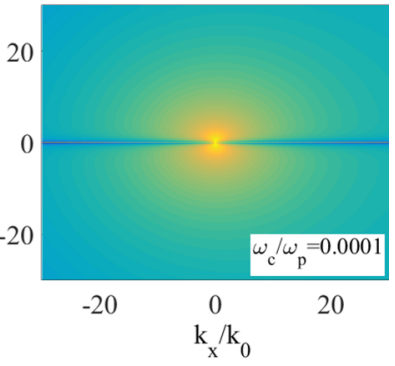

FIG. 4. SPP EFC (density plot) for $\omega_{0}=\omega_{\text {spp }}$ for different values of bias strength. The red arrows indicate the direction of SPP energy flow.

an anisotropic medium the energy flow direction defined by the group velocity $\nabla_{\mathbf{k}} \omega_{\mathbf{k}}$ (orthogonal to the equifrequency contour) does not necessarily coincide with the direction of the plasmon wave vector $\mathbf{k}=\left(k_{x}, k_{y}, 0\right)$. For the case of $\omega_{c} / \omega_{p}=0$ (nonbiased plasma), the EFC is a circle, such that at each point $\left(k_{x}, k_{y}\right)$ on the EFC the flow of energy is along the direction normal to the circle, and thereby the SPP emission is omnidirectional when $\boldsymbol{\gamma}=\gamma \hat{\mathbf{z}}$

For a very weak value of the bias [Fig. 3(a)], the circle becomes slightly elongated along the $k_{x}$ axis. For a larger bias the circle opens up [Fig. 3(b)], and as the bias is increased further, the EFC become hyperbolic-like in the range $\omega_{-}<$ $\omega<\omega_{+}$, leading to a unidirectional (nonreciprocal) SPP beam with energy flow indicated by the red arrows in Fig. 3(c). The SPP far-field pattern at two values of bias is shown in Fig. 3(d), where it can be seen that the SPP forms two narrow beams. The Poynting vector of the short-wavelength SPPs is always directed towards the $x>0$ semi-plane in the interval $\omega_{-}<\omega<\omega_{+}$. Although SPPs on a biased plasma have been long studied (see, e.g., Refs. [49]), this narrow-beam, angle-dependent aspect of the SPP has not been predicted previously.

Remarkably, a strong recoil force can persist even for very small values of the gyration pseudovector (e.g., cyclotron frequency/bias). Figure 1(c) shows the recoil force as a function of magnetic bias and atomic transition frequency, where, for a given cyclotron frequency $\omega_{c}$, the bright areas correspond to $\omega_{0}=\omega_{ \pm}$. Figure 1(d) depicts the recoil force as a function of the cyclotron frequency for different atomic transition frequencies [i.e., three horizontal slices from Fig. 1(c)], showing that for each $\omega_{0}$ there is an optimal value for the bias (vertical dashed line) that maximizes the recoil force $\left[\omega_{0}=\omega_{ \pm}\left(\omega_{c}\right)\right]$. As $\omega_{0} \rightarrow \omega_{\text {spp }}$ an ultrastrong recoil force is obtained for very small bias, as shown in detail in Fig. 2(b).

In order to understand the phenomenon of strong force with small bias, it can be observed that as the bias field approaches zero, the SPP frequency span $\omega_{-}<\omega<\omega_{+}$becomes narrower and narrower, as $\omega_{+}$and $\omega_{-}$approach $\omega_{\text {spp }}$ [see, e.g., Fig. 2(a)], the point at which the two bright lines in Fig. 1(c) intersect. Figure 4 shows the EFC of the corresponding SPP for $\omega_{0}=$ $\omega_{\text {spp }}$ for small plasma bias. For small bias the EFC hyperbola branches become aligned along the $k_{y}$ axis [Fig. 4(a)]. In particular, for Fig. 4(a) there is a wide spectral region near the center of the EFC (roughly $\left|k_{y}\right| / k_{0}<10$ ), for which $k_{x} \neq 0$, and a strong force contribution exists. Thus, the ultrastrong force at a weak bias is due to the high density of states determined by the hyperbolic EFC.

Further support for a strong recoil force at low bias when $\omega_{0} \rightarrow \omega_{\text {spp }}$ comes from the quasistatic solution (6); in the limit of no bias, it can be shown that $\omega_{\theta} \rightarrow \omega_{\text {spp }}+\omega_{c} / 2 \cos \theta$ and $a_{\theta} \rightarrow 1 / 2$. Then, for a $z$-polarized atom $\left(\Gamma_{+, \theta}=1\right),(6)$ becomes

$$
\tilde{\mathrm{F}}_{x}=-\rho_{e e}(t) \frac{\omega_{\mathrm{spp}}}{\omega_{c}} \frac{\omega_{0}-\omega_{\mathrm{spp}}}{\sqrt{\left(\omega_{c} / 2\right)^{2}-\left(\omega_{0}-\omega_{\mathrm{spp}}\right)^{2}}} .
$$

Therefore, the quasistatic force diverges when $\left|\omega_{0}-\omega_{\text {spp }}\right|=$ $\left|\omega_{c}\right| / 2$, i.e., when the atomic transition frequency matches $\omega_{ \pm}$. Although material absorption ensures that the exact force is finite, the divergence of the quasistatic result indicates a strong force persisting as $\omega_{c} \rightarrow 0$. However, below a critical bias value [Fig. 4(b)], the system tends towards isotropic (i.e., $\left.\varepsilon_{g} \ll \varepsilon_{t}\right)$, the interface does not support SPP modes, and the force becomes weak.

Although the presented results apply to the plasma dispersion (4), for a more complicated plasma model, e.g., InSb [40], which includes phonon contributions, the same qualitative behavior is found, although the quasistatic analysis does not lead to simple closed-form expressions. Furthermore, an interface with any Chern-type periodic photonic topological insulator would provide a net spontaneous recoil force due to the unidirectionality of the SPP.

In the absence of an external excitation, the spontaneous emission depopulates the atom, leading to zero steady-state force. To achieve a steady recoil force, the atom can be pumped by a laser. Starting with the master equation for a general nonreciprocal, lossy, and inhomogeneous environment, the atomic population is [43]

$$
\begin{aligned}
\rho_{e e}(t)= & \frac{2 \tilde{\Omega}^{2}}{\lambda_{1} \lambda_{2}}+\frac{\lambda_{1}\left(\lambda_{1}+1 / 2\right)+2 \tilde{\Omega}^{2}}{\lambda_{1}\left(\lambda_{1}-\lambda_{2}\right)} e^{\lambda_{1} \tilde{t}} \\
& +\frac{\lambda_{2}\left(\lambda_{2}+1 / 2\right)+2 \tilde{\Omega}^{2}}{\lambda_{2}\left(\lambda_{2}-\lambda_{1}\right)} e^{\lambda_{2} \tilde{t}},
\end{aligned}
$$

where $\tilde{\Omega}=\Omega / \Gamma$, with $\Omega=\boldsymbol{\gamma} \cdot \mathbf{E} / \hbar$ the Rabi frequency and $\Gamma$ the decay rate, $\tilde{t}=\Gamma t$, and where $\lambda_{1,2}=$ $\frac{1}{2}\left(-3 / 2 \pm \sqrt{1 / 4-16 \tilde{\Omega}^{2}}\right)$. Under steady-state conditions $t \rightarrow \infty, \rho_{e e, s s}=4 \tilde{\Omega}^{2} /\left(1+8 \tilde{\Omega}^{2}\right)$.

Figure 5 shows the normalized force $\tilde{\mathrm{F}}_{x}=\mathcal{F}_{x}^{\text {ss }}(t) / \mathcal{F}_{0}$ as a function time for different laser intensities. When $\Omega / \Gamma=0$ the force decays to zero, whereas for the pumped cases a nonzero steady-state force exists. The laser can be applied orthogonal to the $x$ axis, so as not to influence the net lateral force.

To provide a quantitative estimate of the recoil force, the normalization constant $\mathcal{F}_{0}$ is $0.075 \mathrm{pN}\left(\tilde{D} / \tilde{d}^{4}\right)$, where $\tilde{D}$ is the dipole moment in debye and $\tilde{d}$ is the atom-interface distance in $\mathrm{nm}$. For example, for a Rydberg atom [50] having $\gamma=7900 \mathrm{D}$ located $100 \mathrm{~nm}$ above the substrate, $\mathcal{F}_{0}=0.047 \mathrm{pN}$. Alternatively, a superconducting qubit [51] attached to a cantilever could be used to detect the force. One possible implementation of the plasma is InSb, where $\omega_{p} /(2 \pi) \approx 5 \mathrm{THz}$ for the samples measured in Ref. [40], and $\omega_{c} /(2 \pi)$ ranges from 1 to $6 \mathrm{THz}$ for magnetic bias $1-5 \mathrm{~T}$, respectively. 


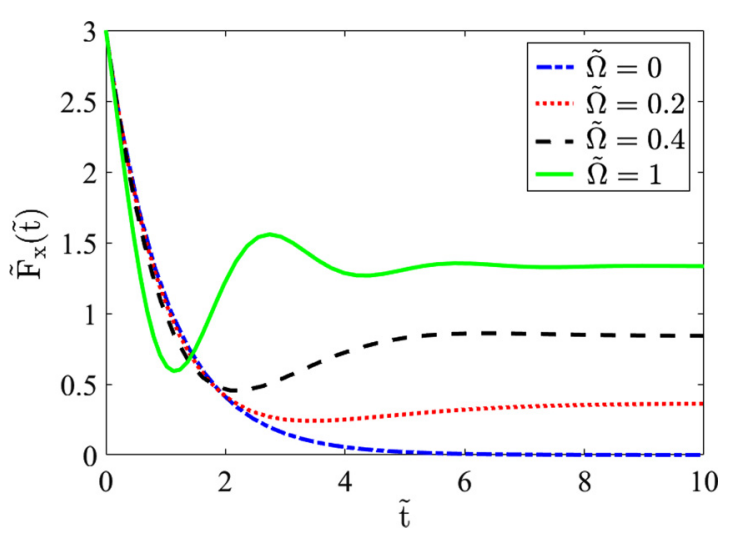

FIG. 5. Normalized recoil force as a function of time for the case of $\omega_{0} / \omega_{p}=0.6, \omega_{c} / \omega_{p}=0.4$ with the atom at $d=0.01 c / \omega_{p}$ above the magnetized plasma in vacuum.

In summary, we derived an exact equation for the spontaneous emission lateral recoil force on an excited atom near an interface with a photonic topological medium. The lateral force emerges in a homogeneous system in a thermal equilibrium, and its sign is independent of the atom polarization and orientation. In order to understand the behavior of the recoil force, the SPPs guided by the interface of the topological medium and vacuum have been investigated. Due to the quasihyperbolic nature of the PTI, the SPPs emitted by an excited atom form narrow beams, whose direction is dependent on the atomic transition frequency. The force is maximized at certain atomic transition frequencies, and persists down to very small bias values due to the high density of photonic states. To achieve a steady recoil force, the quantum dynamics of the atom has been studied by solving the relevant master equation for a laser-pumped system.

The authors gratefully acknowledge discussions with S. Buhmann. This work was partially funded by Fundação para a Ciência e a Tecnologia under Project No. PTDC/EEITEL/4543/2014 and by Instituto de Telecomunicações under Project No. UID/EEA/50008/2013. M.S. thanks the CNRS and the group Theory of Light-Matter and Quantum Phenomena of the Laboratoire Charles Coulomb for hospitality during his stay in Montpellier.
[1] S. Chu, Nobel lecture: The manipulation of neutral particles, Rev. Mod. Phys. 70, 685 (1998).

[2] W. D. Phillips, Nobel lecture: Laser cooling and trapping of neutral atoms, Rev. Mod. Phys. 70, 721 (1998).

[3] C. N. Cohen-Tannoudji, Nobel lecture: Manipulating atoms with photons, Rev. Mod. Phys. 70, 707 (1998).

[4] A. Ashkin, Acceleration and Trapping of Particles by Radiation Pressure, Phys. Rev. Lett. 24, 156 (1970).

[5] J. P. Gordon and A. Ashkin, Motion of atoms in a radiation trap, Phys. Rev. A 21, 1606, (1980).

[6] H. B. G. Casimir, and D. Polder, The influence of retardation on the London-van der Waals forces, Phys. Rev. 73, 360 (1948).

[7] I. E. Dzyaloshinskii, E. M. Lifshitz, and L. P. Pitaevskii, The general theory of van der Waals forces, Adv. Phys. 10, 165 (1961).

[8] M. Antezza, Lev P. Pitaevskii, and S. Stringari, New Asymptotic Behavior of the Surface-Atom Force out of Thermal Equilibrium, Phys. Rev. Lett. 95, 113202 (2005).

[9] J. M. Obrecht, R. J. Wild, M. Antezza, L. P. Pitaevskii, S. Stringari, and E. A. Cornell, Measurement of the Temperature Dependence of the Casimir-Polder Force, Phys. Rev. Lett. 98, 063201 (2007).

[10] M. G. Silveirinha, Casimir interaction between metal-dielectric metamaterial slabs: Attraction at all macroscopic distances, Phys. Rev. B 82, 085101 (2010).

[11] J. N. Munday, F. Capasso, and V. A. Parsegian, Measured longrange repulsive Casimir-Lifshitz forces, Nature (London) 457, 170 (2009).

[12] T. H. Boyer, Van der Waals forces and zero-point energy for dielectric and permeable materials, Phys. Rev. A 9, 2078 (1974).

[13] S. I. Maslovski and M. G. Silveirinha, Mimicking Boyer's Casimir repulsion with a nanowire material, Phys. Rev. A 83, 022508 (2011).
[14] F. Intravaia, C. Henkel, and M. Antezza, Fluctuation-induced forces between atoms and surfaces: The Casimir-Polder interaction, in Casimir Physics, edited by D. Dalvit, P. Milonni, D. Roberts, and F. da Rosa, Lecture Notes in Physics Vol. 834 (Springer, Berlin, 2010).

[15] I. S. Nefedov and J. M. Rubi, Lateral-drag propulsion forces induced by anisotropy, Sci. Rep. 7, 6155 (2017).

[16] S. B. Wang and C. T. Chan, Lateral optical force on chiral particles near a surface, Nat. Commun. 5, 3307 (2014).

[17] F. J. Rodríguez-Fortuño, G. Marino, P. Ginzburg, D. O'Connor, A. Martínez, G. A. Wurtz, and A. V. Zayats, Near-field interference for the unidirectional excitation of electromagnetic guided modes, Science 340, 328 (2013).

[18] F. J. Rodríguez-Fortuño, N. Engheta, A. Martínez, and A. V. Zayats, Lateral forces on circularly polarizable particles near a surface, Nat. Commun. 6, 8799 (2015).

[19] S. Lannebere and M. G. Silveirinha, Negative spontaneous emission by a moving two-level atom, J. Opt. 19, 014004 (2017).

[20] M. G. Silveirinha, Optical Instabilities and Spontaneous Light Emission by Polarizable Moving Matter, Phys. Rev. X 4, 031013 (2014).

[21] F. D. M. Haldane and S. Raghu, Possible Realization of Directional Optical Waveguides in Photonic Crystals with Broken Time-Reversal Symmetry, Phys. Rev. Lett. 100, 013904 (2008).

[22] S. Raghu and F. D. M. Haldane, Analogs of quantum-Hall-effect edge states in photonic crystals, Phys. Rev. A 78, 033834 (2008).

[23] Z. Wang, Y. Chong, J. D. Joannopoulos, and M. Soljačić, Observation of unidirectional backscattering immune topological electromagnetic states, Nature (London) 461, 772 (2009).

[24] L. Lu, J. D. Joannopoulos, and M. Soljacic, Topological photonics, Nat. Photonics 8, 821 (2014). 
[25] L. Lu, J. D. Joannopoulos, and M. Soljacic, Topological states in photonic systems, Nat. Phys. 12, 626 (2016).

[26] M. G. Silveirinha, Chern invariants for continuous media, Phys. Rev. B 92, 125153 (2015).

[27] M. G. Silveirinha, Bulk-edge correspondence for topological photonic continua, Phys. Rev. B 94, 205105 (2016).

[28] S. A. H. Gangaraj, M. G. Silveirinha, and G. W. Hanson, Berry phase, Berry connection, and Chern number for a continuum bianisotropic material from a classical electromagnetics perspective, IEEE J. Multiscale Multiphys. Comput. Technol. 2, 3 (2017).

[29] B. Yang, M. Lawrence, W. Gao, Q. Guo, and S. Zhang, Oneway helical electromagnetic wave propagation supported by magnetized plasma, Sci. Rep. 6, 21461 (2016).

[30] A. R. Davoyan and N. Engheta, Theory of Wave Propagation in Magnetized Near-Zero-Epsilon Metamaterials: Evidence for One-Way Photonic States and Magnetically Switched Transparency and Opacity, Phys. Rev. Lett. 111, 257401 (2013).

[31] D. Jin, L. Lu, Z. Wang, C. Fang, J. D. Joannopoulos, M. Soljacic, L. Fu, and N. X. Fang, Topological magnetoplasmon, Nat. Commun. 7, 13486 (2016).

[32] M. G. Silveirinha, Topological angular momentum and radiative heat transport in closed orbits, Phys. Rev. B 95, 115103 (2017).

[33] S. Fuchs, F. Lindel, R. V. Krems, G. W. Hanson, M. Antezza, and S. Buhmann, Casimir-Lifshitz force for nonreciprocal media and applications to photonic topological insulators, Phys. Rev. A 96, 062505 (2017).

[34] S. A. Hassani Gangaraj, G. W. Hanson, and M. Antezza, Robust entanglement with 3D nonreciprocal photonic topological insulators, Phys. Rev. A 95, 063807 (2017).

[35] S. Scheel, S. Y. Buhmann, C. Clausen, and P. Schneeweiss, Directional spontaneous emission and lateral Casimir-Polder force on an atom close to a nanofiber, Phys. Rev. A 92, 043819 (2015).

[36] M. G. Silveirinha, S. A. H. Gangaraj, G. W. Hanson, and M. Antezza, Fluctuation-induced forces on an atom near a photonic topological material, Phys. Rev. A 97, 022509 (2018).

[37] S. Y. Buhmann, L. Knoll, D.-G. Welsch, and H. T. Dung, Casimir-Polder forces: A nonperturbative approach, Phys. Rev. A 70, 052117 (2004).
[38] S. Y. Buhmann and S. Scheel, Thermal Casimir versus CasimirPolder Forces: Equilibrium and Nonequilibrium Forces, Phys. Rev. Lett. 100, 253201 (2008).

[39] S. A. H. Gangaraj, A. Nemilentsau, and G. W. Hanson, The effects of three-dimensional defects on one-way surface plasmon propagation for photonic topological insulators comprised of continuum media, Sci. Rep. 6, 30055 (2016).

[40] E. Palik, R. Kaplan, R. Gammon, H. Kaplan, R. Wallis, and J. Quinn, Coupled surface magnetoplasmon-optic-phonon polariton modes on InSb, Phys. Rev. B 13, 2497 (1976).

[41] E. Moncada-Villa, V. Fernandez-Hurtado, F. J. Garcia-Vidal, A Garcia-Martin, and J. C. Cuevas, Magnetic field control of nearfield radiative heat transfer and the realization of highly tunable hyperbolic thermal emitters, Phys. Rev. B 92, 125418 (2015).

[42] J. A. Bittencourt, Fundamentals of Plasma Physics, 3rd ed. (Springer, New York, 2010).

[43] See Supplemental Material at http://link.aps.org/supplemental/ 10.1103/PhysRevB.97.201108 for a description of the optical force parameters and a detailed calculation of the quantum master equation for the dynamics of a single emitter in terms of the exact system Green function.

[44] R. N. C. Pfeifer, T. A. Nieminen, N. R. Heckenberg, and H. Rubinsztein-Dunlop, Momentum of an electromagnetic wave in dielectric media, Rev. Mod. Phys. 79, 1197 (2007).

[45] S. M. Barnett, Resolution of the Abraham-Minkowski Dilemma, Phys. Rev. Lett. 104, 070401 (2010).

[46] S. M. Barnett and R. Loudon, The enigma of optical momentum in a medium, Philos. Trans. R. Soc., A 368, 927 (2010).

[47] M. G. Silveirinha, Reexamination of the Abraham-Minkowski dilemma, Phys. Rev. A 96, 033831 (2017).

[48] K. Y. Bliokh, A. Y. Bekshaev, and F. Nori, Optical Momentum, Spin, and Angular Momentum in Dispersive Media, Phys. Rev. Lett. 119, 073901 (2017).

[49] S. R. Seshadri, Excitation of surface waves on a perfectly conducting screen covered with anisotropic plasma, IRE Trans. Microwave Theory Tech. 10, 573 (1962).

[50] T. F. Gallagher, Rydberg atoms, Rep. Prog. Phys. 51, 143 (1988).

[51] O. Astafiev, A. M. Zagoskin, A. A. Abdumalikov, Jr., Yu. A. Pashkin, T. Yamamoto, K. Inomata, Y. Nakamura, and J. S. Tsai, Resonance fluorescence of a single artificial atom, Science $\mathbf{3 2 7}$ 840 (2010). 